\title{
Liposomal doxorubicin extravasation controlled by phenotype-specific transport properties of tumor microenvironment and vascular barrier
}

\author{
Kenji Yokoi ${ }^{1}$, Diana Chan ${ }^{1}$, Milos Kojic ${ }^{1,2,3}$, Miljan Milosevic ${ }^{1,2}$, David Engler ${ }^{1}$, Rise Matsunami ${ }^{1}$, \\ Tomonori Tanei $^{1}$, Yuki Saito ${ }^{1}$, Mauro Ferrari ${ }^{1}$, Arturas Ziemys ${ }^{1 *}$ \\ ${ }^{1}$ The Houston Methodist Research Institute, Houston, TX 77030; ${ }^{2}$ Belgrade Metropolitan \\ University, Research and Development Center for Bioengineering, 3400 Kragujevac, Serbia; ${ }^{3}$ \\ Serbian Academy of Sciences and Arts, 11000 Belgrade, Serbia. \\ *Corresponding author: aziemys@houstonmethodist.org; (713) 441-7320
}

Keywords: kinetics, nanotherapeutics, drug vector, liposome, doxorubicin, computational diffusion model

\begin{abstract}
Although nanotherapeutics can be advantageous over free chemotherapy, the benefits of drug vectors can vary from patient to patient based on differences in tumor microenvironments. Although systemic pharmacokinetics (PK) of drugs is considered as the major determinant of its efficacy in clinics, recent clinical and basic research indicates that tumor-based PK can provide better representation of therapeutic efficacy. Here, we have studied the role of the tumor extravascular tissue in the extravasation kinetics of doxorubicin (DOX), delivered by pegylated liposomes (PLD), to murine lung (3LL) and breast (4T1) tumors. We found that phenotypically different 3LL and 4T1 tumors shared the similar systemic PK, but DOX extravasation in the tumor extravascular tissue was substantially different. Liquid chromatography-mass spectrometry (LC-MS) measurements showed that DOX fluorescence imaged by fluorescence microscopy could be used as a marker to study tumor microenvironment PK, providing an excellent match to DOX kinetics in tumor tissues. Our results also suggest that therapeutic responses can be closely related to the interplay of concentration levels and exposure times in extravascular tissue of tumors. Finally, the computational model of capillary drug transport showed that internalization of drug vectors was critical and could lead to 2-3 orders of magnitude more efficient drug delivery into the extravascular tissue, compared to noninternalized localization of drug vectors, and explaining the differences in therapeutic efficacy between the $3 \mathrm{LL}$ and $4 \mathrm{~T} 1$ tumors. These results show that drug transport and partitioning characteristics can be phenotype- and microenvironment-dependent and are highly important in drug delivery and therapeutic efficacy.
\end{abstract}

\section{Introduction}

Interest in biomedical nanoparticle and microparticle applications is growing, especially for imaging and drug delivery [1-3]. Rapid advances in nanotechnology have enabled incorporation 
of chemotherapeutics into nanoparticles for delivery of a large payload [4,5]. There are more than 30 drug delivery technologies in clinics or clinical trials, where the majority are liposomal formulations [6]; many more are in active development. Pegylated liposomal doxorubicin (PLD) is a liposomal formulation of doxorubicin (DOX) and is among the best known examples of nanotherapeutics. PLD has reduced uptake by the reticulo-endothelial system due to the attached polyethylene glycol polymers and stably retains the drug by entrapment inside the nanoparticle. These features result in pharmacokinetics (PK) with dramatically extended circulation time and a reduced volume of distribution, as compared to those of free DOX [7]. Cardiotoxicity, which is a major adverse effect of free DOX, is significantly reduced in the patients treated with PLD. Thus, preferential accumulation to tumor utilizing the enhanced permeability and retention (EPR) effect, extended systemic PK profile with reduced systemic adverse events made PLD as one of the most widely used nanotherapeutics approved in Europe for the treatment of various types of tumors, including metastatic breast and lung cancer [8].

Nevertheless, survival rates in patients with metastatic tumors has not been significantly improved for more than a decade [9]. The benefits of nanotherapeutics may vary from patient to patient based on differences in the tumor and organ microenvironment $[10,11]$, like: abnormal and heterogeneous tumor blood perfusion and extravasation of therapeutics, extracellular matrix blockage of penetration of therapeutics, or efflux pumps expressed on various cancer cells which can discharge anticancer drugs and downgrade pharmacodynamics (PD). Nevertheless, poor drug delivery and limited tumor uptake is often neglected as a mechanism of drug resistance in cancer [12].

Although the systemic pharmacokinetics (PK) of drugs has been thought to be a major determinant of utility and efficacy in the clinic, recent evidences indicate that systemic PK may not be indicative of its therapeutic efficacy and does not reflect intra- and inter-tumor heterogeneity $[13,14]$. PD studies of 5-fluorouracil (5-FU) show that there is an excellent association between tumor-based PK and response [15]. Our recent study using PLD demonstrated that the amount of collagen of type-IV in basal membrane of capillaries in tumor can determine the extravasation of DOX [16]. The in vivo and computational studies suggested that murine lung ( $3 \mathrm{LL}$ ) tumors have more collagen around capillaries as compared to murine breast (4T1) tumor, which could have prevented efficient PLD transport and limited DOX transport into tumor extravascular tissue.

It is therefore important to have adequate knowledge of the mechanisms which determine the transport of drugs from the systemic circulation to tumor sites [17]. Thus, the meaningful tumor microenvironments effects on PK should be studied to improve therapeutic efficacy. Here, we have studied the phenotypic specificity of PK of the 3LL and 4T1 tumors by investigating DOX delivery by PLD, which serves as an example to other nanotherapeutics, as well.

\section{Materials and methods}

Cells. The 4T1 murine breast cancer and 3LL murine lung cancer cells were kindly provided by Dr. Isaiah J. Fidler (University of Texas MD Anderson Cancer Center, Houston, TX, USA). The 
cells were maintained in minimal essential medium supplemented with $10 \%$ fetal bovine serum and supplements, as described previously [18].

Mice. Female BALB/c and C57/BL6 mice were maintained in animal facilities at Houston Methodist Research Institute and care was approved by the American Association for Accreditation of Laboratory Animal Care and in accordance with current regulations and standards of the United States Department of Agriculture, Department of Health and Human Services, and the National Institutes of Health.

Establishment of experimental tumors. To produce tumors, 4T1 and 3LL cells were harvested from subconfluent cultures. A tumor cell suspension $\left(1 \times 10^{5}\right.$ cells $\left./ 100 \mu \mathrm{l}\right)$ was injected subcutaneously into BALB/C or C57/BL6 mice.

Therapy and necropsy. Starting 10 days after the inoculation with the tumor cells, the mice were intravenously injected with $6 \mathrm{mg} / \mathrm{kg}$ of PLD (Doxoves ${ }^{\mathrm{TM}}$-Liposomal Doxorubicin $\mathrm{HCl}$; FormuMax Scientific Inc., Palo Alto, CA, USA) and the mice were sacrificed 24 hours after the injection. Then, the tumors were excised, frozen, and stored for subsequent evaluation of endothelial cells, basement membranes, and the accumulation of PLD in the tumors.

Immunofluorescent staining of endothelial cells (CD31) and imaging of PLD in the tumors. The frozen sections were fixed in cold acetone for 15 minutes. After protein blocking, immunofluorescent staining of the endothelial cells or basement membrane was performed using antibodies to CD31 (BD Biosciences, San Jose, CA, USA) [18]. The sections were then incubated with the corresponding fluorochrome-labeled secondary antibodies (Jackson Immuno Research, West Grove, PA, USA). The images were captured using a confocal laser scanning microscope (Carl Zeiss Microlmaging Inc., Thornwood, NY, USA) and analyzed using the built-in image analysis software [18]. The ruby red fluorescence of anthracycline in the tumor tissue was also imaged using confocal microscopy at the excitation and emission wavelengths of $488 \mathrm{~nm}$ and $590 \mathrm{~nm}$, respectively [19, 20]. The imaged fluorescence of DOX originated mostly from free DOX, because the solid state of DOX inside the PLD strongly quenched the fluorescence of liposomal DOX [21].

LC-MS. DOX measurements were carried out on a Waters NanoAcquity/Xevo TQ ultraperformance liquid chromatography tandem mass spectrometry (UPLC/MS/MS) system utilizing a LC/MRM methodology. Samples were extracted by organic precipitation and loaded onto a C-18 column; gradient LC separation was accomplished with a water/acetonitrile/formic acid buffer system, and ions injected into the mass spectrometer using positive ion electrospray conditions. Daunorubicin was used as an internal standard.

Imaging analysis. The ImageJ program was used to analyze the tissue samples [22] using the adequate RGB channels as 8-bit images, which were treated with background subtraction and automatic thresholding using the triangle method. The results presented in this study were calculated from five replicate measurements. The whole images were used in the analysis, and the numbers of collagen and vessel entities after the automatic triangle thresholding were calculated using a standard particle analysis procedure. To avoid misinterpreting monocytes as vessels in the CD31 staining, the minimal area was set to $250 \mu \mathrm{m}^{2}$. The colocalization between collagen and vessels was calculated by using the ImageJ/Fiji program (coloc2 plug-in) [23], where the M1 coefficient denoted the overlap of vessels by collagen, and M2 denoted the 
overlap of collagen by vessels. The extravasation of the dextran tracer and DOX was calculated as a function of the distance in terms of pixels (after automatic thresholding). In the case of dextran, the distance was calculated to the nearest vessel, whereas in the case of DOX, the distance was calculated to the centers of red cell nuclei. The cell counts (DOX-affected and proliferating cells stained with Ki67) and their statistics were calculated by using stained-nuclei centers.

Drug transport model. We applied a recently developed, hierarchical, multiscale diffusion modeling technique [24-27], which has an advantage over other approaches because it accounted for the polymer microstructure and the physicochemical characteristics of the diffusing molecules, including the size of the molecules or particles. See Supplementary Material for details.

\section{Results}

To evaluate DOX kinetics in the 3LL and 4T1 tumor microenvironments, in vivo experiments were conducted using a single PLD dose, and DOX concentration changes were measured at 0 , $4,24,48,72$, and 120 hours. Plasma, bulk tumor, and tumor microenvironments were quantified and correlated by DOX concentrations. A DOX transport model was applied to analyze the DOX kinetics in in tumor extravascular tissue.

3.1. Systemic PK. DOX concentrations were measured by using LC-MS for tumors and plasma. The plasma concentration of DOX decreased exponentially, approaching undetectable concentrations at 48 hours after DOX administration (Figure 1A). The concentration profiles were fit best using a two-exponent fitting analysis, suggesting that DOX clearance was affected by a slower-equilibrating tissue compartment. DOX concentration profiles in tumors, measured using a bulk resected volume, revealed that the 4T1 tumor had an extended profile, while the $3 \mathrm{LL}$ tumor had a profile resembling the plasma. The concentrations in the tumors were 20-30 times lower than in plasma. DOX concentration remained at detectible levels inside tumors even after 120 hours, especially in the 4T1 tumors, while DOX was eliminated from the plasma after 48 hours in both tumor types. 

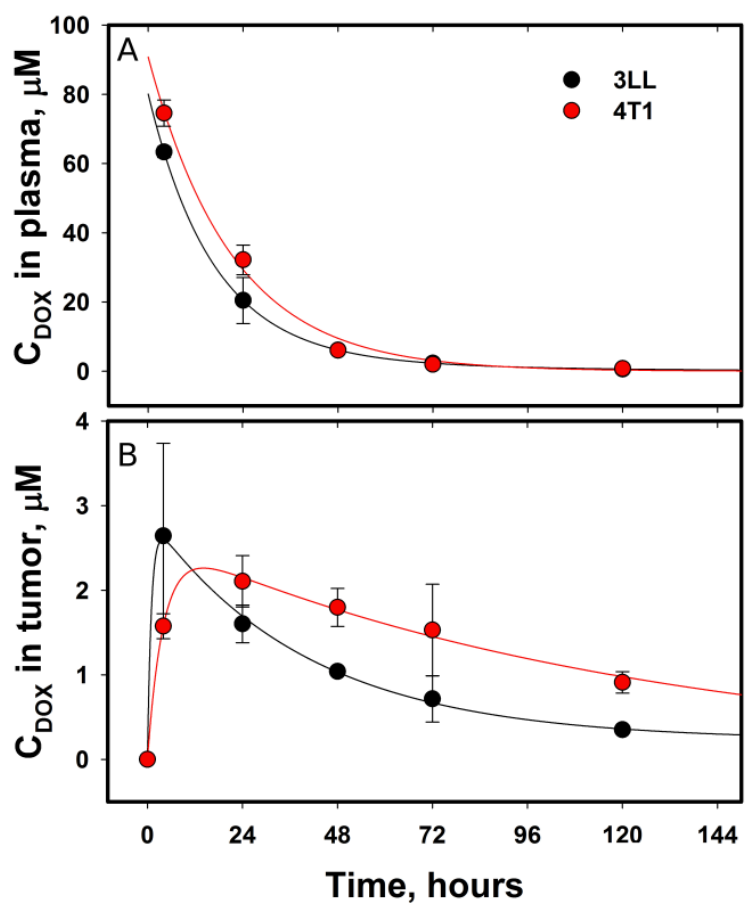

Figure 1. Concentration profiles of DOX in plasma (A) and in the 3LL and 4T1 tumor tissues (B) after a single PLD injection at a dose of $6 \mathrm{mg} / \mathrm{kg}$ of DOX.

3.2. Quantification using fluorescence. To correlate DOX concentrations in tumor extravascular tissue with the 4T1 and 3LL cell cultures, cells were incubated with DOX. After 24 hours, cell cultures were imaged using the same protocol as the tumor pathology slices (Figure $2 \mathrm{~A}-\mathrm{B}$ ) and DOX concentrations were quantified using LC-MS. The dependence of the measured DOX concentration inside the 3LL and 4T1 cells on the DOX concentration in the incubation media was exponential (Figure 2C), where DOX concentrations inside cells were lower by one order of magnitude compared to DOX concentrations in the incubation media. The $4 \mathrm{~T} 1$ cells showed lower DOX accumulation than the $3 \mathrm{LL}$ cells. The standard curves were constructed relating DOX fluorescence in cells with DOX concentration, which was then used to quantify the histology samples (Figure 2D). Autofluorescence background was found in both cell types, thus limiting DOX quantification below $0.01 \mu \mathrm{M}$ and $0.2 \mu \mathrm{M}$ in the 4T1 and 3LL cells, respectively. The standard curves were linear above those critical concentrations and fitted with the following equations:

$$
\begin{array}{lll}
\text { 3LL: } & C_{D O X}=0.4226 * \operatorname{Int}-0.3561 & \left(R^{2}=0.90\right) \\
\text { 4T1: } & C_{D O X}=0.0503 * \operatorname{Int}-0.1305 & \left(R^{2}=0.98\right)
\end{array}
$$

As it is shown in Figure 2D and according the Beer-Lambert law, DOX fluorescence in the 3LL cells had a higher molar emission compared to that of the 4T1 cells. DOX concentration was evaluated inside histology slices of both tumors, as it is illustrated in Figure 2E-F. DOX 
quantification revealed almost no DOX extravasation into extravascular tissue in the $3 \mathrm{LL}$ tumors with very high DOX accumulation within areas of few vessels (Figure 2E). DOX extravasation was higher in the 4T1 tumors, showing high DOX concentrations (and fluorescence) in areas of vessels and around them. Using this approach, DOX concentration was quantified at all in vivo time points.
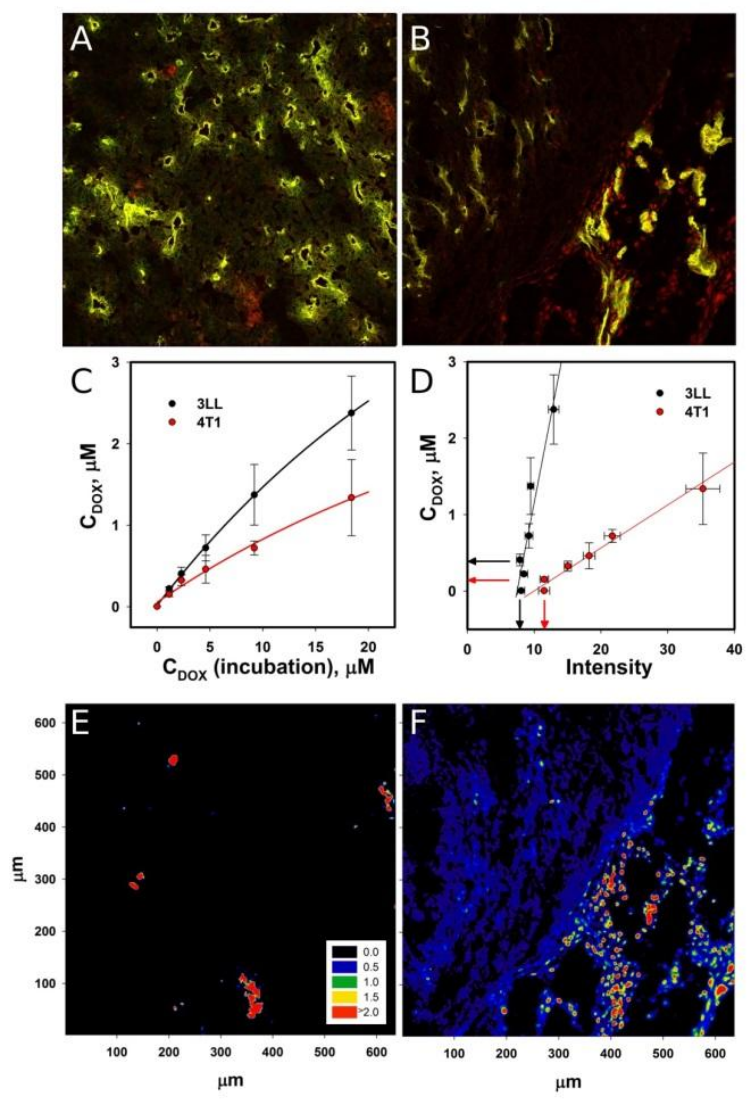

Figure 2. DOX quantification in the microenvironment of the $3 L L$ and $4 T 1$ tumors. The representative $3 \mathrm{LL}(\mathbf{A})$ and $4 \mathrm{~T} 1$ (B) tumors depicting the CD31 (green) immunostained capillaries and the extravasation of DOX (red) at 24 hours; the colocalization of CD31 and DOX fluorescence resulted in yellow. In vitro $3 \mathrm{LL}$ and $4 \mathrm{~T} 1$ cell cultures were incubated in DOX for 24 hours and DOX concentration in cells was measured by LC-MS (C). The DOX fluorescence in cells was correlated to the DOX concentrations (D); the arrows at the axes mark concentration and intensity regions, where DOX concentration can be predicted based on tissue autofluorescence. The DOX concentration maps of the 3LL and 4T1 tumors ( $E$ and $F$ ) based on DOX fluorescence in A-B and correlation in $\mathbf{D}$.

3.3. DOX kinetics inside tumor extravascular tissue. Subtracting the plasma component from the DOX concentration in a tumor will estimate DOX concentration in the tumor extravascular tissue. The estimated plasma volume fraction in tumors was approximately $1.8 \%$ [28]. Our pathology analysis showed that the area fraction of stained endothelia was almost two times lower in the $3 \mathrm{LL}$ tumors, and the plasma fraction was reduced twice for the $3 \mathrm{LL}$ tumor samples (see Supplementary Material). The correction of DOX concentration in the tumor did not 
change the profile of the 3LL tumors, but shifted the DOX concentration peak substantially from 14 to 45 hours in the $4 \mathrm{~T} 1$ tumors (Figure $3 \mathrm{~A}$ ).

DOX concentration profiles were calculated from histological samples using the approach described above. Figure 3B shows DOX concentrations calculated from fluorescence imaging by LC-MS and compares the concentration kinetics to the measured concentration for the 4T1 tumors. The estimated DOX concentrations from the histological samples matched well the concentration profiles in tumor extravascular tissue, as measured by LC-MS. The histology data did not correlate with DOX concentrations in plasma and bulk tumor tissues. The 3LL tumor, however, did not show any detectable DOX outside the vessels in the tumor tissue; therefore, there was no way to perform a similar correlation analysis.

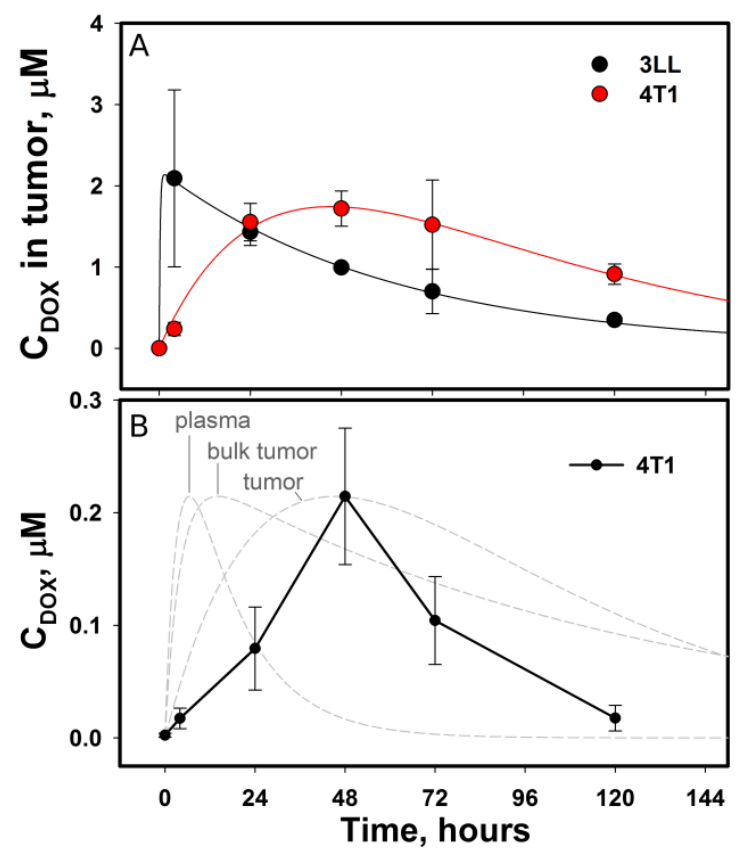

Figure 3. Correlation between systemic and microenvironment concentration kinetics of DOX. The tumor concentration was adjusted by subtracting an estimated capillary plasma volume and providing DOX concentration kinetics in the tumor extravascular tissue(A). DOX concentration kinetics, which was estimated using fluorescence, correlated ideally with the adjusted DOX concentration in the tumor extravascular tissue(gray dashed lines; scaled for comparison) (B).

Table 1. The pharmacokinetic characteristics of DOX $\left(C_{\max }-\right.$ maximum concentration, $t_{\max }-$ the time of $C_{\max }, t_{1 / 2, A}$ - the half-life of absorption, $t_{1 / 2, E}$ - the half-life of elimination, AUC - the area under the curve).

\begin{tabular}{lllllll} 
& Plasma & \multicolumn{3}{c}{ Tumor (bulk) } & \multicolumn{2}{c}{ Tumor (corrected) } \\
\hline & $3 \mathrm{LL}$ & $4 \mathrm{~T} 1$ & $3 \mathrm{LL}$ & $4 \mathrm{~T} 1$ & $3 \mathrm{LL}$ & $4 \mathrm{~T} 1$ \\
\hline $\mathrm{t}_{\max }[$ hours $]$ & 2.3 & 6.5 & 3.9 & 14.1 & 2.4 & 45.2 \\
$\mathrm{C}_{\max }[\mu \mathrm{M}]$ & 80.1 & 90.8 & 2.4 & 2.3 & 2.1 & 1.7
\end{tabular}




$\begin{array}{lllllll}\mathrm{t}_{1 / 2, \mathrm{~A}} \text { [hours] } & - & - & 0.3 & 3.0 & 0.4 & 10.2 \\ \mathrm{t}_{1 / 2, \mathrm{E}}[\text { hours] } & 15.5 & 20.5 & 36.6 & 102.6 & 45.2 & 124.0 \\ \mathrm{AUC}[\mu \mathrm{M} \cdot \text { hours] } & 1431.8 & 1945.9 & 137.8 & 306.6 & 134.1 & 217.7\end{array}$

Table 1 shows the main PK parameters quantifying DOX kinetics. DOX concentration in the 3LL tumor was very similar to plasma. However, DOX concentration and retention were enhanced in the 4T1 tumors, especially in the tumor extravascular tissue (without plasma): the elimination half-life $\left(t_{1 / 2, E}\right)$, the area under the curve (AUC), and the time of maximum concentration were increased.

The DOX distribution kinetics was evaluated in tumor extravascular tissue to provide mechanistic aspects of DOX pharmacokinetics in tumor microenvironments. Quantified DOX extravasation away from vessels showed that the average DOX concentration for all vessels found in the investigated tumor slices gradually increased and then decreased, with a peak at 48 hours (Figure 4A). Interestingly, the DOX extravasation depth from vessels remained similar at all time points and did not exceed $50 \mu \mathrm{m}$. We hypothesized that DOX mass gets sequestered by its absorption into nuclei of cells adjacent to capillaries; a similar hypothesis about the role of drug absorption was verbalized in Ref. [29], too. Therefore, we employed our computational mass transport analysis to simulate mass transport in capillaries, relying on physical properties such as diffusion and drug partitioning. Simulation results showed that increasing absorption, or preferential drug partitioning into cells, reduced drug extravasation depth (Figure 4B-D). The majority of drug was sequestered into cells (Figure 4E) and the fraction of free-to-diffuse drug was depleted, which could impair drug diffusion deeper into extravascular tissues. The results of our model collaborate with our in vivo findings. 

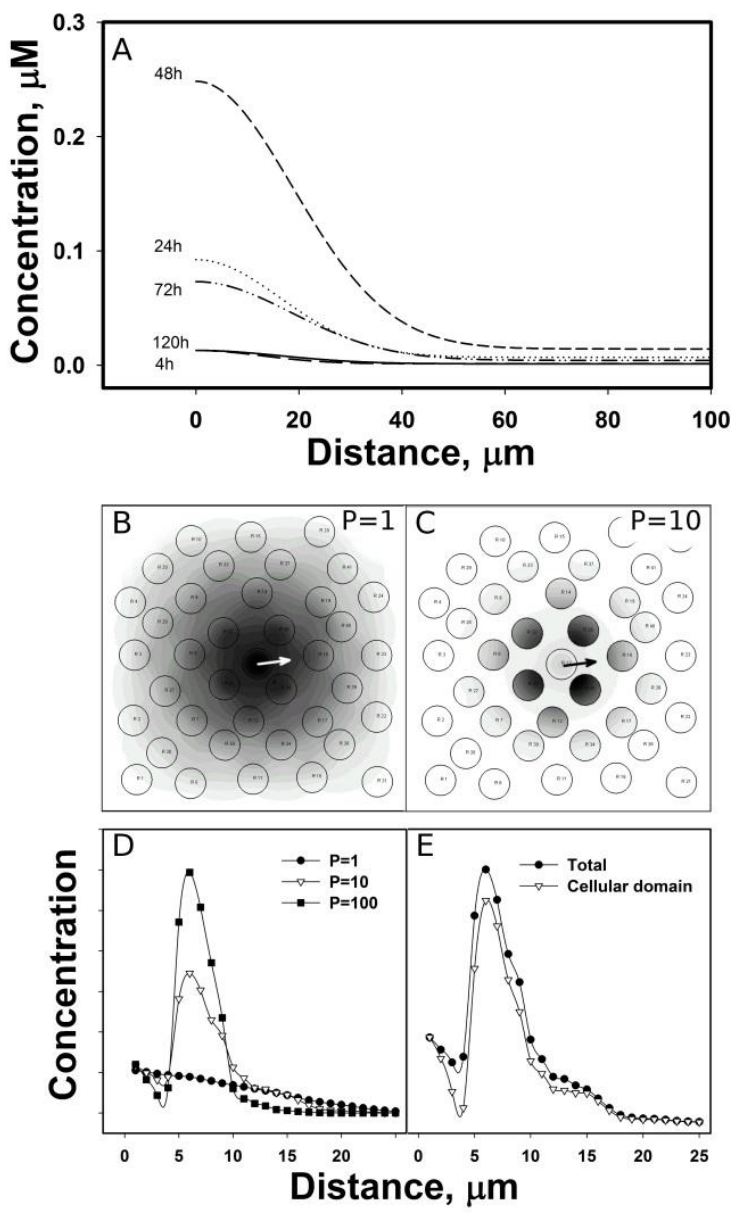

Figure 4. DOX extravasation in the tumor extravascular tissue. The quantified DOX extravasation from vessels, using PLD delivery, was up to $50 \mu \mathrm{m}$ at every time point and was independent of concentration (A). The snapshots of drug concentration fields in the extravascular tissue after diffusion from a vessel (arrows) were simulated by the capillary transport model without $(B)$ and with $(C)$ molecular partitioning $(P)$ into the microenvironment of cells. The drug extravasation depth decreased by increasing drug partitioning (or absorption) (D), leading to drug accumulating closer to vessels inside cellular compartments at larger P (E; P =10); this accumulation, in turn, decreased the free-to-diffuse fraction of the drug.

3.4. Zone of influence (ZOI). To quantify the efficiency of DOX extravasation into the 4T1 tumor extravascular tissue, the ZOI - the concept first introduced in [30] - was calculated using the area fraction occupied with a certain critical or higher concentration in histology slices by using fluorescence analysis. Figure 5A shows time evolutions of ZOI for DOX concentrations of $1 \mu \mathrm{M}$ and $0.1 \mu \mathrm{M}$ calculated by using fluorescence. Both profiles have maxima at 48 hours, but differ in the fractions: with $0.1 \mu \mathrm{M}$ occupying approximately $60 \%$ of the tumor slice area, and $1 \mu \mathrm{M}-$ only $1 \%$ of the area. High concentration localize around vessels, while low - at vessel peripheries (Figure 4A). 

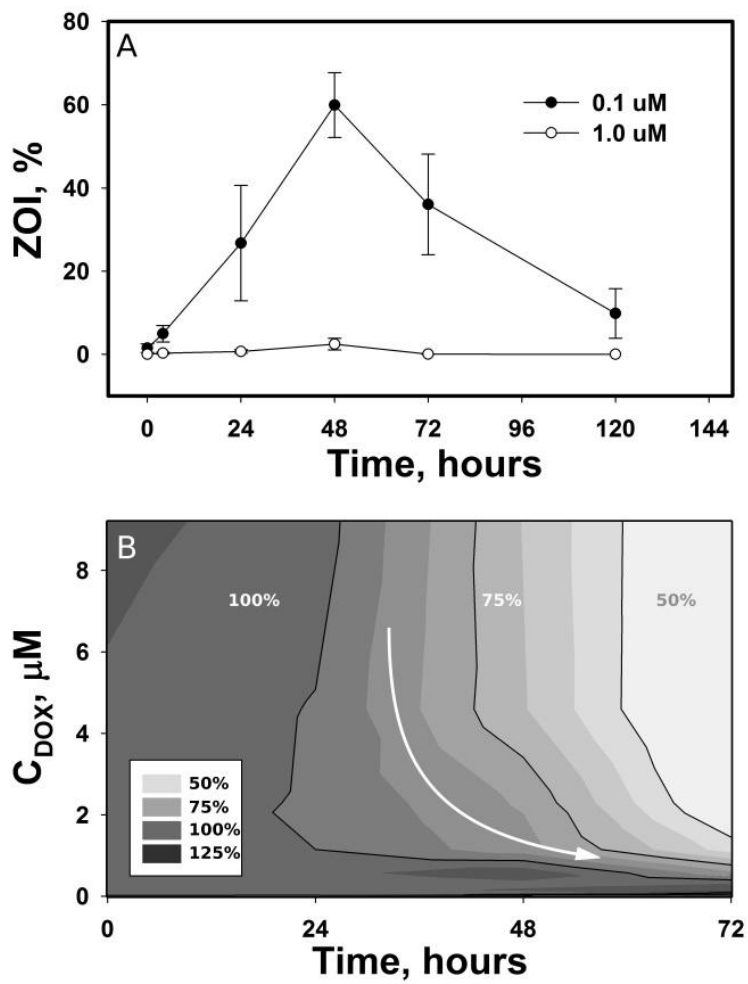

Figure 5. The time evolution of the zone of influence (ZOI) as the area fraction of DOX above 1 and $0.1 \mu \mathrm{M}$, based on fluorescence imaging $(\mathbf{A})$. The $4 \mathrm{~T} 1$ cell cultures as a function of DOX concentration and time (B). Results show that smaller concentrations may require longer times (white arrow) to cause biological effects that can be predicted by analyzing ZOI and DOX kinetics inside the tumor extravascular tissue.

To establish the relationship of concentration and ZOI, we tested 4T1 cell viability as a function of time and DOX concentration (Figure 5B). The results showed that cell viability had the same dependence on time at DOX concentrations above $4 \mu \mathrm{M}$; however below $4 \mu \mathrm{M}$, cell viability was time dependent. At a DOX concentration below $1 \mu \mathrm{M}, 3-5$ days may be required to show an effect on cell viability. Therefore, the zones of concentrations in tumor extravascular tissue could dictate the therapeutic outcome by relating local concentration and exposure time in microenvironment. These in vitro results may not fully represent how DOX concentrations affect in the actual tumor cells in in vivo, but similar concentration-time effects should be expected inside the tumors as well. 


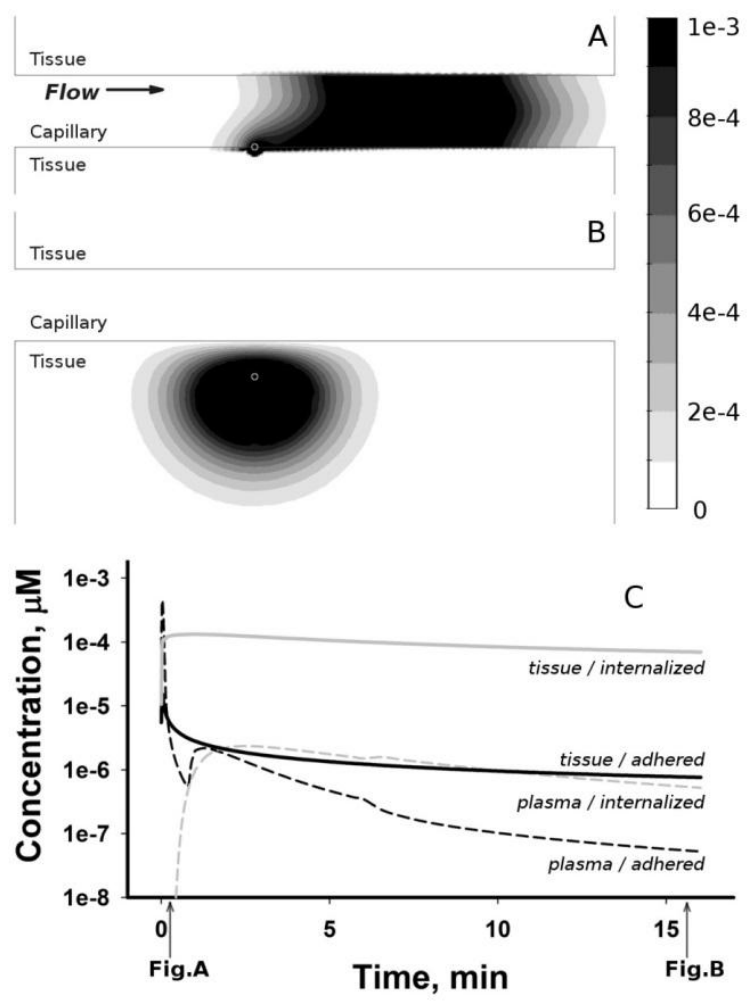

Figure 6. Calculated DOX concentration pharmacokinetics in the tumor extravascular tissue, by using the computational capillary model. The association of PLD on a vessel wall (A) and deeper internalization of PLD (B) led to different calculated concentration profiles in the tumor extravascular tissue and plasma compartments. The model recapitulated DOX concentration profiles in the 3LL and 4T1 tumors (C) and suggested that the 4T1 tumor had internalized PLD. Conversely, the $3 \mathrm{LL}$ tumor contained PLD mostly confined inside vessels. The arrows show the time-points corresponding to the extravasation fields in $\mathbf{A}$ and $\mathbf{B}$.

The computational capillary model further showed how the final localization of a drug vector affected $\mathrm{ZOI}$ and concentration kinetics inside the tumor extravascular tissue. Figure $6 \mathrm{~A}$ and $\mathrm{B}$ depicts the drug concentration field release from a liposome-like drug vector adhered onto the capillary wall and internalized into the extravascular tissue. As shown, the concentration of the adhering vector was rapidly cleared by blood flow, unless it was internalized. Figure 6C shows the kinetics of mean concentration in plasma and extravascular compartments. According to simulated results, drug concentration in the tumor extravascular compartment can be 2-3 orders of magnitude higher for an internalized vector compared to vectors adhering onto vessel walls and exposed to the plasma flow.

\section{Discussion}

We have determined DOX concentration kinetics to establish a relationship between tumor microenvironments and DOX extravasation efficiency using nanotherapeutics. The systemic PK of DOX concentration in plasma was very similar between the 3LL and 4T1 tumor phenotypes 
which have shown similar sensitivity to PLD in vitro [18]. Nevertheless, our previous and current results show that therapeutic outcomes can be fundamentally different for those tumors and favoring the survival of mice bearing the 4T1 tumors [31]. One of the reasons behind why the 4T1 is sensitive to PLD is micro-pharmacokinetics ( $\mu \mathrm{PK}$ ) of DOX in microenvironment, specifically - tumor extravascular tissue. The computational capillary models have shown that the location of a drug vector, PLD in this study, could dictate how efficiently the payload penetrated into the tumor extravascular tissue. The internalization of PLD into the tumor extravascular tissueensured efficient drug extravasation, while the association of a drug vector onto vessel walls provided only very marginal benefits in drug extravasation. Our current in vivo and in silico results, together with our previous study on vascular collagen in the 3LL and 4T1 tumors [16], suggest that PLD gets internalized deeper into the tumor extravascular tissue space of the 4T1 tumors; which is not a case for 3LL. Deeper DOX penetration into extravascular tissueleads to larger ZOI, which could be associate with the potency of payload delivery, and shorter times required to exert pharmacological effect. The computational models based on physical principals of transport provide especially well illustrated example that the endpoint of drug vectors in extravascular compartment may dramatically modulate drug ZOI and the efficiency of delivery. Moreover, the similarity of DOX concentration kinetics in tumors with plasma kinetics of the $3 \mathrm{LL}$ tumors supported the assumption that PLD could be only associated with vessel walls on the plasma side. The later collaborated well with Figure 2E showing that PLD in the 3LL tumors can be found in some cases only confined within vessel areas of pathology slices.

DOX fluorescence had an excellent correlation with the DOX concentration measured in the tumor extravascular tissue. Using both techniques, DOX concentration was highest at 48 hours in the 4T1 tumors. The peak of DOX extravasation at 48 hours coincided with our anti-vascular drug studies, showing that PLD damaged the endothelia of capillaries, making them leaky with a peak at 48 hours, followed by vascular healing (K.Yokoi; unpublished, manuscript in preparation). Other studies have also shown that PLD may cause a breakdown of tumor vasculature [32]. Therefore, we conclude that the most efficient DOX extravasation is associated with the damage of vascular barrier.

DOX fluorescence after 3-4 days was almost undetectable, while LC-MS detected DOX concentrations at levels of $1 \mu \mathrm{M}$ even after 5 days, suggesting the existence of a large, but not fluorescent DOX pool. The DOX concentration evaluated from DOX fluorescence and LC-MS/MS revealed that the concentration of DOX measured by fluorescence was approximately five times lower in the tumor extravascular tissuethan that measured by LC-MS in the 4T1 tumors, which is very similar to DOX concentration differences in in vitro studies of fluorescence (Figure 2C). Several factors might contribute to this difference, including differences in fluorescence quenching between in vitro and in vivo experiments, and the presence of microenvironment in in vivo studies. The error in the standard curves for the 4T1 cells was up to $20-35 \%$ of most and cannot alone explain the difference. It is known that tetracycline fluorescence can be quenched by its strong binding, changes in surrounding media, $\mathrm{pH}$, and $\mathrm{CO}_{2}$ levels $[33,34]$. The binding constants of DOX to DNA can be up to $10^{8}$ with a residual fluorescence of $2-40 \%[35,36]$, meaning that bound DOX can be fluorescently less apparent then free DOX. Based on these results we could hypothesize that there is phenotype-specific 
DOX binding inside cells and that pathology imaging can not provide precise measurement of concentrations, although can be used as an excellent marker of local $\mu \mathrm{PK}$.

Finally, our results help to analyze DOX transport in extravasation. The peak of DOX extravasation in 4T1 tumors occurred long after the injection allowing for sufficient time to extravasate. However, the peak of DOX extravasation remained around $50 \mu \mathrm{m}$ of tumor capillaries at all time points: initial, maximum, and decline phases of DOX concentrations. The concentration peak at 48 hours almost tripled the concentration found at 24 hours. Other study found a comparable penetration depth of DOX in other tumors after 24 hours [37], but kinetics was missing. Relying on classical diffusion theory (Fick's laws), the higher gradient of DOX should promote DOX penetration farther, especially after so many hours, but this was not observed. We hypothesized that DOX was absorbed or bound inside the tumor extravascular tissue, preventing its diffusion deeper. Our computational drug transport analysis confirmed that DOX absorption into cells was a physical process that depleted the fraction of free DOX that was capable of diffusing deeper into the extravascular space. Independently, a similar assumption was made in the study of drug penetration into colon carcinoma spheroids, demonstrating that more binding led to less penetration [29].

\section{Concluding remarks}

The results suggest that transport properties of the tumor microenvironment and vascular barrier are critical in efficient drug delivery of nanotherapeutics. Our experimental and computational models showed that systemic PK may not always be an adequate tool to study nanotherapeutics, because it did not provide any differentiation between the two tumor models with good and no DOX extravasation. DOX, as well as any other therapeutic payloads, could persist in the tumor and its microenvironment long after measurable drug levels were cleared from the plasma compartment and could still provide therapeutic effects. For the same reason liposomal formulation - and other drug vectors as well - are perhaps more effective treating cancer compared to free drugs. DOX has tendency to provide anti-vascular effect enhancing its extravasation, but strong DOX absorption into cells of extravascular tissuestill limits its extravasation at $50 \mu \mathrm{m}$. The interplay of concentrations and exposure times to DOX, as well as the fraction of tumor affected by therapeutically significant DOX concentrations may be critical for better efficacy. In view of these results, tumor microenvironment pharmacokinetics and its transport properties should get more emphasis in drug delivery, because microenvironment PK can be the critical chain explaining therapeutic efficacy of nanotherapeutics and other formulations of anticancer drugs. 


\section{Acknowledgments}

This project was partially supported by the Houston Methodist Research Institute and grant OI 174028 of the Serbian Ministry of Education and Science. The authors also acknowledge partial support from the following funding sources: the National Institutes of Health (U54CA143837 to M.F. and K.Y.; U54CA151668 to M.F.), the Ernest Cockrell Jr. Distinguished Endowed Chair (to M.F.), and the US Department of Defense (W81XWH-09-1-0212 to M.F.). The authors acknowledge the Texas Advanced Computing Center (TACC) at The University of Texas at Austin for providing HPC resources that contributed to the results reported in this paper.

\section{References}

1. Koo, O.M., I. Rubinstein, and H. Onyuksel, Role of nanotechnology in targeted drug delivery and imaging: a concise review. Nanomedicine: Nanotechnology, Biology and Medicine, 2005. 1(3): p. 193-212.

2. Ferrari, M., Cancer nanotechnology: opportunities and challenges. Nat Rev Cancer, 2005. 5(3): p. 161--171.

3. Peer, D., et al., Nanocarriers as an emerging platform for cancer therapy. Nature Nanotechnology, 2007. 2(12): p. 751-760.

4. Ferrari, M., Nanogeometry: beyond drug delivery. Nature Nanotechnology, 2008. 3(3): p. 131132.

5. Wang, A.Z., R. Langer, and O.C. Farokhzad, Nanoparticle delivery of cancer drugs. Annual Review of Medicine, 2012. 63: p. 185-198.

6. Norvaisas, P. and A. Ziemys, The Role of Payload Hydrophobicity in Nanotherapeutic Pharmacokinetics. Journal of Pharmaceutical Sciences, 2014.

7. Gabizon, A., H. Shmeeda, and Y. Barenholz, Pharmacokinetics of pegylated liposomal doxorubicin. Clinical Pharmacokinetics, 2003. 42(5): p. 419-436.

8. Duggan, S.T. and G.M. Keating, Pegylated Liposomal Doxorubicin. Drugs, 2011. 71(18): p. 25312558.

9. Freyer, G., et al., Pharmacokinetic studies in cancer chemotherapy: usefulness in clinical practice. Cancer Treatment Reviews, 1997. 23(3): p. 153.

10. Maeda, H., et al., Tumor vascular permeability and the EPR effect in macromolecular therapeutics: a review. Journal of Controlled Release, 2000. 65(1): p. 271-284.

11. Davis, M.E., Nanoparticle therapeutics: an emerging treatment modality for cancer. Nature Reviews Drug Discovery, 2008. 7(9): p. 771-782.

12. Minchinton, A.I. and I.F. Tannock, Drug penetration in solid tumours. Nature Reviews Cancer, 2006. 6(8): p. 583-592.

13. Wolf, W. and C.A. Presant, Tumor-based pharmacokinetics has greater significance for anticancer drugs than does blood-based pharmacokinetics. Clinical Pharmacology \& Therapeutics, 2004. 76(5): p. 508-508.

14. Müller, M., A. dela Peña, and H. Derendorf, Issues in pharmacokinetics and pharmacodynamics of anti-infective agents: distribution in tissue. Antimicrobial Agents and Chemotherapy, 2004. 48(5): p. 1441-1453.

15. Presant, C., et al., Association of intratumoral pharmacokinetics of fluorouracil with clinical response. The Lancet, 1994. 343(8907): p. 1184-1187.

16. Yokoi, K., et al., Capillary-wall collagen as a biophysical marker of nanotherapeutic permeability into the tumor microenvironment. Cancer Research, 2014. 74(16): p. 4239-4246. 
17. McVie, J.G., The role of pharmacokinetics in (combination) chemotherapy. Cancer, 1984. 54(S1): p. 1175-1178.

18. Yokoi, K., et al., Porous silicon nanocarriers for dual targeting tumor associated endothelial cells and macrophages in stroma of orthotopic human pancreatic cancers. Cancer Letters, 2012.

19. Vaage, J., et al., Tumour uptake of doxorubicin in polyethylene glycol-coated liposomes and therapeutic effect against a xenografted human pancreatic carcinoma. British Journal of Cancer, 1997. 75(4): p. 482.

20. Erikson, A., et al., The impact of enzymatic degradation on the uptake of differently sized therapeutic molecules. Anticancer Research, 2008. 28(6A): p. 3557-3566.

21. Lee, R.J. and P.S. Low, Folate-mediated tumor cell targeting of liposome-entrapped doxorubicin in vitro. Biochimica et Biophysica Acta (BBA)-Biomembranes, 1995. 1233(2): p. 134-144.

22. Abràmoff, M.D., P.J. Magalhães, and S.J. Ram, Image processing with ImageJ. Biophotonics international, 2004. 11(7): p. 36-42.

23. Schindelin, J., et al., Fiji: an open-source platform for biological-image analysis. Nature Methods, 2012. 9(7): p. 676-682.

24. Ziemys, A., et al., Hierarchical modeling of diffusive transport through nanochannels by coupling molecular dynamics with finite element method. Journal of Computational Physics, 2011. 230(14): p. 5722-5731.

25. Kojic, M., et al., On diffusion in nanospace. Journal of the Serbian Society for Computational Mechanics, 2011. 5(1): p. 104-118.

26. Kojic, M., et al., Transport in biological systems. Journal of the Serbian Society for Computational Mechanics/Vol, 2011. 5(2): p. 101-128.

27. Kojic, M., et al., A multiscale MD-FE model of diffusion in composite media with internal surface interaction based on numerical homogenization procedure. Computer Methods in Applied Mechanics and Engineering, 2014. 269: p. 123-138.

28. Pathak, A.P., et al., MR-derived cerebral blood volume maps: Issues regarding histological validation and assessment of tumor angiogenesis. Magnetic Resonance in Medicine, 2001. 46(4): p. 735-747.

29. Erlanson, M., E. Daniel-Szolgay, and J. Carlsson, Relations between the penetration, binding and average concentration of cytostatic drugs in human tumour spheroids. Cancer Chemotherapy and Pharmacology, 1992. 29(5): p. 343-353.

30. Ziemys, A., et al., Computational analysis of drug transport in tumor microenvironment as a critical compartment for nanotherapeutic pharmacokinetics. Drug Delivery, 2015. (accepted)(DRD-2015-0021.R1).

31. Yokoi, K., et al., Serum biomarkers for personalization of nanotherapeutics-based therapy in different tumor and organ microenvironments. Cancer Letters, 2014. 345(1): p. 48-55.

32. Zhou, R., R. Mazurchuk, and R.M. Straubinger, Antivasculature effects of doxorubicin-containing liposomes in an intracranial rat brain tumor model. Cancer Research, 2002. 62(9): p. 2561-2566.

33. Karukstis, K.K., et al., Deciphering the fluorescence signature of daunomycin and doxorubicin. Biophysical Chemistry, 1998. 73(3): p. 249-263.

34. Simon, S., D. RoY, and M. Schindler, Intracellular $\mathrm{pH}$ and the control of multidrug resistance. Proceedings of the National Academy of Sciences, 1994. 91(3): p. 1128-1132.

35. Roche, C.J., J.A. Thomson, and D.M. Crothers, Site selectivity of daunomycin. Biochemistry, 1994. 33(4): p. 926-935.

36. Rapoport, N. and L. Pitina, Intracellular distribution and intracellular dynamics of a spin-labeled analogue of doxorubicin by fluorescence and EPR spectroscopy. Journal of Pharmaceutical Sciences, 1998. 87(3): p. 321-325. 
37. Primeau, A.J., et al., The distribution of the anticancer drug Doxorubicin in relation to blood vessels in solid tumors. Clinical Cancer Research, 2005. 11(24): p. 8782-8788. 
Phenotype differences in transport

Non-internalyzed drug vector

Internalyzed drug vector

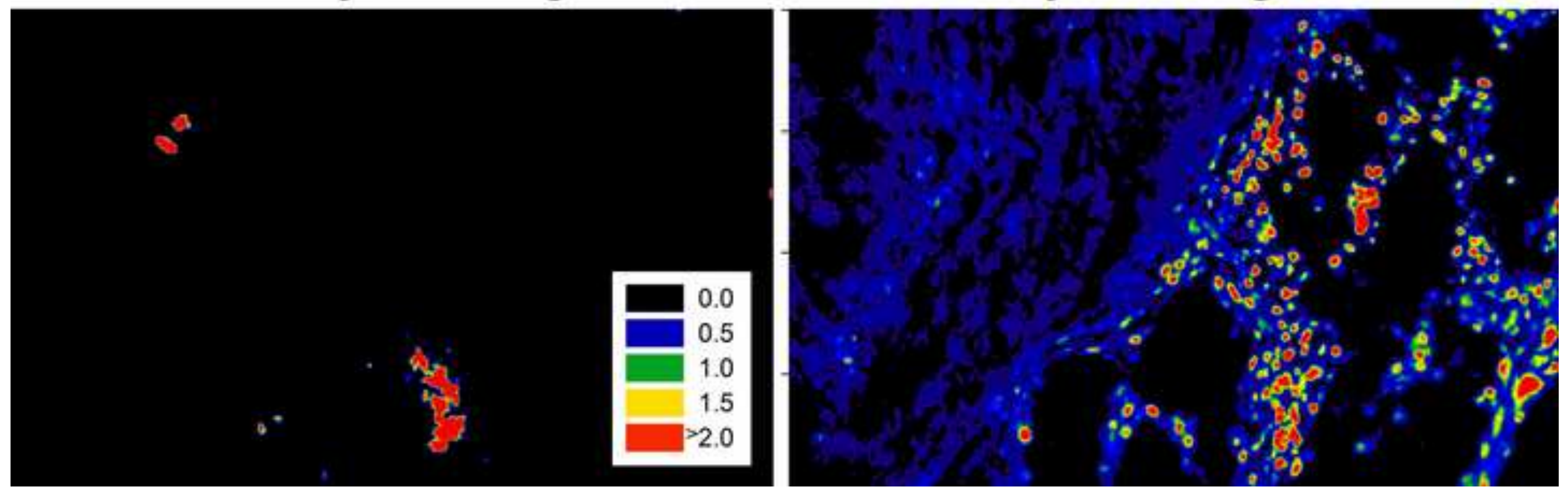

